\section{Covid-19 pandemic and pediatric healthcare policy in Italy: time for a change}

\author{
Biagio Solarino, ${ }^{1}$ Maurizio Aricò ${ }^{2}$ \\ 1 Interdisciplinary Department of \\ Medicine, University of Bari - Section of \\ Legal Medicine, Bari General Hospital, \\ Bari; ${ }^{2}$ Azienda Ospedaliero \\ Universitaria Consorziale Policlinico, \\ Giovanni XXIII Children Hospital, Bari, \\ Italy
}

\begin{abstract}
The outbreak of coronavirus disease 2019 pandemic forced the Italian Government to act extreme measures that put the entire country under lockdown. The citizens experimented a radical transformation of their lives while the healthcare system started building a novel COVID-system that quickly dissolved the former organization, included pediatric healthcare network. For a number of reasons COVID-19 pandemic is polite with children; however, the throw into the COVID activities, all outpatient practices were virtually abolished (or at least sharply reduced): programmed admission were postponed, and elective surgery was limited to very selected cases requiring urgent oncologic treatment, trauma or emergency. Primary care visits were reduced, and soon thereafter prohibited, trying to support the patients with remote (phone, WhatsApp or videocall) aid. The healthcare system we have been working in simply dissolved within days.
\end{abstract} fear of this unknown disease drove to develop dedicated pathways in the Pediatric Emergency Departments for the suspected and diagnosed cases of COVID-19, together with their relatives. The combination of conflicting feelings resulted in an unexpected scenario: the number of accesses to the pediatric Hospital or Unit(s) sharply dropped to less than half. How do we explain this phenomenon? The authors believe that our health care system may learn a lesson from the catastrophe of COVID, if we will be able to redesign our way of providing our cares to children. Nothing should be taken for granted, because this might be a real "time for a change".

\section{Editorial}

The outbreak of coronavirus disease 2019 (COVID-19) pandemic induced a radical transformation in our lives. Italy rapidly turned out to be one of the most heavily affected countries worldwide. ${ }^{1}$ Thus, the top priority of the policymakers became to reduce the pandemic morbidity and mortality rates as much as possible. With the aim of reducing the R0 index to lower than 1.0, the government followed the example set by China to impose "social distancing". 2 This resulted in extreme measures that put the entire country under lockdown. The leading hypothesis was that an explosive spike of COVID-19 dissemination would have been unbearable for the Italian healthcare system, especially in terms of number of Intensive Care Unit (ICU) beds and facilities simultaneously available. Thus, while the people was practically locked down at home (or at the site they happened to be), the healthcare system(s) started building a novel, parallel COVID-system: many hospitals, usually the largest and more advances ones, were either converted into "COVID-19 hospital" or split into a COVID-area and a residual, minor nonCOVID area. With the double aim of reducing the circulation of patients in the hospital area, but also to spare human resources to

\section{COVID and children care}

For a number of reasons, not yet fully clarified, COVID-19 pandemic is polite with children. ${ }^{3}$ Symptoms caused by this coronavirus are similar to those regularly observed in winter season in children with common viral infections; the risk to develop serious complications is minimal, and the fatality rate is almost absent. 4

Yet, pediatric healthcare network was also disrupted. The fear of this unknown disease, and uncertainty about the presenting phenotype, drove the need to develop dedicated pathways, as to isolate suspected and diagnosed cases of COVID-19, together with their relatives. A combination of conflicting feelings resulted in an unexpected scenario: despite the rising fear for this new disease, the number of accesses to the Pediatric Emergency Department (pED) sharply drop to less than half. Social distancing measures, leading to closure of schools, parks, gyms and other social places, make the children to spend the entire day indoor, at home, thus reducing not only the risk to be infected by other children with common infectious agents, but also the risk of traumatic injuries. Furthermore, the fear to be infected in the hospital waiting room has likely contributed to reduce the access to the pED. Is this enough to explain this phenomenon? Alternatively, is Covid-19
Correspondence: Biagio Solarino, Interdisciplinary Department of Medicine, University of Bari, Section of legal Medicine, Azienda Ospedaliero Universitaria Consorziale Policlinico, Bari, Italy

E-mail: biagio.solarino@uniba.it

Key words: COVID-19, Pediatric emergency department, healthcare policy, children care.

Contributions: The authors contributed equally.

Conflict of interest: The authors declare no potential conflict of interest.

Received for publication: 3 August 2020. Accepted for publication: 3 August 2020.

This work is licensed under a Creative Commons Attribution NonCommercial 4.0 License (CC BY-NC 4.0).

(C) Copyright: the Author(s), 2020

Licensee PAGEPress, Italy

Pediatric Reports 2020; 12:8823

doi:10.4081/pr.2020.8823

Pandemic disclosing the real need for hospital medical care in Italy? Current child healthcare system comprises three main levels of intervention: a) primary care; 2) secondary care in Pediatric Hospital Units; 3 ) tertiary pediatric children's hospital. ${ }^{5} \mathrm{ED}$ services are provided for free by the National Health System, with only a small co-payment based on family income, which may vary on a regional basis.

COVID-19 pandemic: which lesson to be learned in the organization of public health care system?

In a national survey of $\mathrm{pED}$ activities, Longhi et al. underlined a significantly higher percentage of children hospitalized after accessing the ED in southern regions. ${ }^{6}$ These observations support the concept that the organization of our health care system, although universally accessible regardless the patient/family income, remains someway heterogeneous. COVID-19 pandemic put under the spotlight some inappropriate ways of using it, which cause reduced efficiency and effectiveness. Due to the process of institutional devolution, individual Regions apply different policies. Indeed, we have no more a single NHS, but rather over 20 autonomous regional authorities. Whether this was effective and convenient is today deeply challenged, or even rejected by those who strongly recommend its return to a unique NHS.7 Devolution has been justified by the aim at economies and better administration by managers located closer to the activities. However, is it only a matter 
of money and resources invested? Coming to pediatric health care, during COVID pandemic the access to pediatric ED sharply dropped to one-half. This happened nationwide and within days. Were those missing requests necessary or simply inappropriate? Reasons may rest on both sides, the patient and the doctor. Until the age of 6 years, pediatric primary care is provided during the weekdays only by "family" pediatricians that work on private practice; between 6 and 16 years, one can choose to associate or substitute the pediatrician with a general practitioner. During the weekend, a pediatric ambulatory consulting service located in the hospitals provides primary, nonurgent care. This setting should cover any child need, unless an urgent or even emergency care is needed, requiring admission to EDs, with a yellow or red code, in accordance to pediatric triage scale categorization. 8 Monitoring pediatric EDs before COVID pandemic had clearly documented frequent overcrowding, resulting from inappropriate accesses for children deserving only primary care treatment. ${ }^{9}$

Unnecessary overcrowding in the ED not only increased the waiting time, but also induced frequent complaining by the families. Minimal or low priority (white or green) codes may frequently happen to wait many hours before completing the visits and the exams prescribed (how appropriately?) even for minor disease/symptoms. Sometimes complaining progressed even to assault the ED workers, both doctors and nurses. ${ }^{10,11}$ In this environment, the relationship between doctor and patient/family slips down to a very poor quality, making it difficult for healthcare professional to provide respectful and sensitive care, and create the usual, expected relationship with patient/family. ${ }^{12}$ This may increase the risk of errors and unintended harm. As a reaction to this stressing (or even aggressive) atmosphere, despite moderate or minimal morbidity, EDs pediatrician have frequently hospitalized these children, and prescribed unnecessary (and potentially damaging) imaging studies, in the well-known pattern of "defensive medicine".13,14 Furthermore, also in Italy, like in many other developed countries, health workers (and even hospital administrators) are increasingly exposed to malpractice claims: thus, inappropriate hospitalizations may result from the attempt to defend from any parental claim. Frequently the child caregiver reports insufficient or unsatisfactory phone contact with the family pediatrician, thus resulting in taking the child to the Hospital. If so, the attempt to organize a primary care setting able to prevent the child/family from an unnecessary hospital access has failed. This was the pic- ture most frequently observed nationwide.

In the era of pandemic, emergency regulation prevented family pediatricians from visiting children, thus limiting their role to remote counselling. Yet, instead of further pushing the children toward the hospital, this was associated with a sharp reduction of the hospital access to pED. The parents quickly shifted to a conservative pattern, limiting hospital access to what is perceived as a real emergency. So, what? On one side, one can take the impression that most of unnecessary access have been spared. On the other side, we should consider that inappropriate self-triage made by the parents and driven by pandemic scare may occasionally expose some children to a delayed diagnosis and treatment of severe conditions. 15

In our countries, the evolution of pandemic pushes each of us to think how to go beyond this tragic phase, and how to "reopen Italy". This applies not only to economy and production, but also to public services including the health system. We should be able to invest this "still time" in designing a new model of for pediatric healthcare delivery. We need to be more effective in spending our huge resources, and in particular, to be more appropriate in the choice of the pathways for care of patients with different needs, from the healthy newborn to patients with rare disease and severe handicap, which may impact on the society even more than pediatric cancer. 16 We should be able to rebuild the outpatient network, which may support preventive medicine, early diagnosis and rehabilitation. Fancy technologies, becoming one of the clues to get out of COVID pandemic, may become part of the health-care system, reducing undue transportation by increasing remote control of chronic patients. ${ }^{17}$

In conclusion, we should try to revisit COVID-19 pandemic chronicle and decode the many messages this tragic "natural experiment" provides us. We have learned a lot on the mechanisms underlying the immune system by the tragedy of congenital, once invariably fatal, childhood immune deficiencies. ${ }^{18}$ Comparably, our health care system may learn a lesson from the catastrophe of COVID, if we will be able to redesign our way of providing our cares to children. Nothing should be taken for granted, because this might be a real "time for a change".

\section{References}

1. Armocida B, Formenti B, Ussai S, et al. The Italian health system and the
COVID-19 challenge. Lancet Public Health 2020;5:S2468-67.

2. Hellewell J, Abbott S, Gimma A, et al. Feasibility of controlling COVID-19 outbreaks by isolation of cases and contacts. Lancet Glob Health 2020;8:48896.

3. Caselli D, Aricò M. 2019-nCoV: Polite with children! Pediatr Rep 2020;11: 8495.

4. Ludvigsson JF. Systematic review of COVID-19 in children shows milder cases and a better prognosis than adults. Acta Paediatr 2020;109:1088-95.

5. Corsello G, Ferrara P, Chiamenti G, et al. The Child Health Care System in Italy. J Pediatr 2016;177S:116-26.

6. Longhi R, Picchi R, Minasi D, Di Cesare Merlone A. Pediatric emergency room activities in Italy: a national survey. Ital J Pediatr 2015;41:77-84

7. Mauro M, Maresso A, Guglielmo A..Health decentralization at a deadend: towards new recovery plans for Italian hospitals. Health Policy 2017;121:582-7

8. Riva B, Clavenna A, Cartabia M, et al. Emergency department use by pediatric patients in Lombardy Region, Italy: a population study. BMJ Pediatrics Open 2018;2;000247.

9. Wolfe I, Thompson M, Gill P, et al. Health services for children in western Europe. Lancet 2013;381:1224-34

10. Berlanda S, Pedrazza M, Fraizzoli M, de Cordova F. Addressing Risks of Violence against Healthcare Staff in Emergency Departments: The Effects of Job Satisfaction and Attachment Style. Biomed Res Int 2019; 28:1-12.

11. Benini F, Congedi S, Rossin S, Pennella A. The PIPER WEEKEND study. Children's and adults satisfaction regarding pediatric pain in Italian Emergency Department Ann Ist Super Sanità 2018;54:12-9.

12. Mueller BU, Neuspiel DR, Stucky Fisher ER, et al. Principles of Pediatric Patient Safety: Reducing Harm Due to Medical Care. Pediatrics 2019;143:e20183649.

13. McGrew PR, Chestovich PJ, Fisher JD, et al. Implementation of a CT scan practice guideline for pediatric trauma patients reduces unnecessary scans without impacting outcomes. J Trauma Acute Care Surg 2018;85:451-58

14. Bester JC. Defensive practice is indefensible: how defensive medicine runs counter to the ethical and professional obligations of clinicians. Med Health Care Philos 2020 Apr 17. doi: 10.1007/s11019-020-09950-7. [Epub ahead of print] PMID: 32303983 
15. Lazzerini M, Barbi E, Apicella A, et al. Delayed access or provision of care in Italy resulting from fear of COVID-19. Lancet Child Adolesc Health 2020;4:e10-e11.

16. Campanella P, Azzolini E, Izzi A, et al.
Hospital efficiency: how to spend less maintaining quality? Ann Ist Super Sanita 2017;53:46-53.

17. Hollander JE, Carr BG. Virtually Perfect? Telemedicine for Covid-19. N Engl J Med 2020;382:1679-81.
18. Rubin Z, Pappalardo A, Schwartz A, Antoon JW. Prevalence and Outcomes of Primary Immunodeficiency in Hospitalized Children in the United States. J Allergy Clin Immunol Pract 2018;6:1705-10. 